\title{
A New Instrument for Determining Charge to Diameter Distributions of Charged Particles
}

\author{
James H. Anderson \\ SUNY Empire State College, Genesee Valley Learning Center, 680 Westfall Rd.. Rochester, NY 14620, USA \\ Email: Jim.Anderson@esc.edu
}

\begin{abstract}
A new instrument for measuring the charge-to-diameter ratio of small particles $(2-100 \mu \mathrm{m})$ is described. In this instrument, the terminal velocity of charged particles in an electric field in air is determined by measuring the time of flight of the particles between two laser beams. The main advantages of this instrument are that it is easy to operate, and only about $15 \mathrm{~min}$. are required to obtain a distribution of charge-to-diameter ratio for a few thousand particles. The major disadvantage is that low charged and uncharged particles cannot be detected.
\end{abstract}

Keywords: Particle charge, charge distribution, q/d distribution, triboelectricty, tribocharging, elecrophotographic toners.

\section{Introduction}

One of the most important factors in the reliable operation of elecrophotographic copiers and printers is controlling the electric charge on the toner particles. In two component elecrophotographic developers toner particles charging results from the triboelectric interaction between the toner particles, which form the image and carrier particles, which are retained in the printer. Toner particles are typically made of a polymer material (e.g. polyester) filled with a pigment and often containing small concentrations of additives (e.g. quaternary ammonium salts) to control the charge. In addition, the surface of toner particles is often coated with fine oxide particles (e.g. silica) to control flow and adhesion of the particles. This surface treatment may also have important effects on the particle charge.

Particle charge is most often reported as the average charge per mass $(\mathrm{q} / \mathrm{m})$ of the particles. Many workers have also found it useful to determine the distribution of charges per particle in a population of toner particles or the distribution of charge per diameter $(\mathrm{q} / \mathrm{d})$ of the particles.

Several instruments are commercially available which can determine the distribution of charge to diameter ratio or the joint distribution of particle size and charge of charged toner particles. The Epping $\mathrm{q} / \mathrm{d}$ meter is based on the determination of the terminal velocity of charged particles in an electric field. Details of the design and operation of this instrument are described in $[1,2]$. Charged particles pass through an electric field created by a pair of non-parallel plates and are deposited on a glass slide. The slide is optically scanned to determine the relative concentration of particles as a function of their charge-to-mass ratio. An example of the application of this instrument to polyamide particles used in selective laser sintering is given in [3].

The E-SPART analyzer is capable of determining both the charge and mass distributions of particles simultaneously. Particles pass through an analyzer with crossed electric and acoustic fields. Particle motion is measured using a laser Doppler technique. From the motion of the particles in the acoustic and electric fields, the particle mass and charge can be determined independently. Details of the design and operation of the E-SPART analyzer are given in [4] and [5]. An example of the application of this instrument to particle size and charge distributions of pharmaceutical aerosols generated by inhalers is given in [6].

G.P. Gee, L. B. Schein, C. I. Dodds, and K. A. Tran also describe an instrument based on an earlier design by R. B. Lewis, R. F. Koehler and E. W. Connors [7]. In this instrument particles moving through a chamber by laminar air flow are deflected by an electric field imposed on a pair of parallel plates. The deflection of the particles and their size distribution are determined by an automatic image analysis system. Thus this instrument is capable of determining the charge and size distribution of the particles independently [8]. 


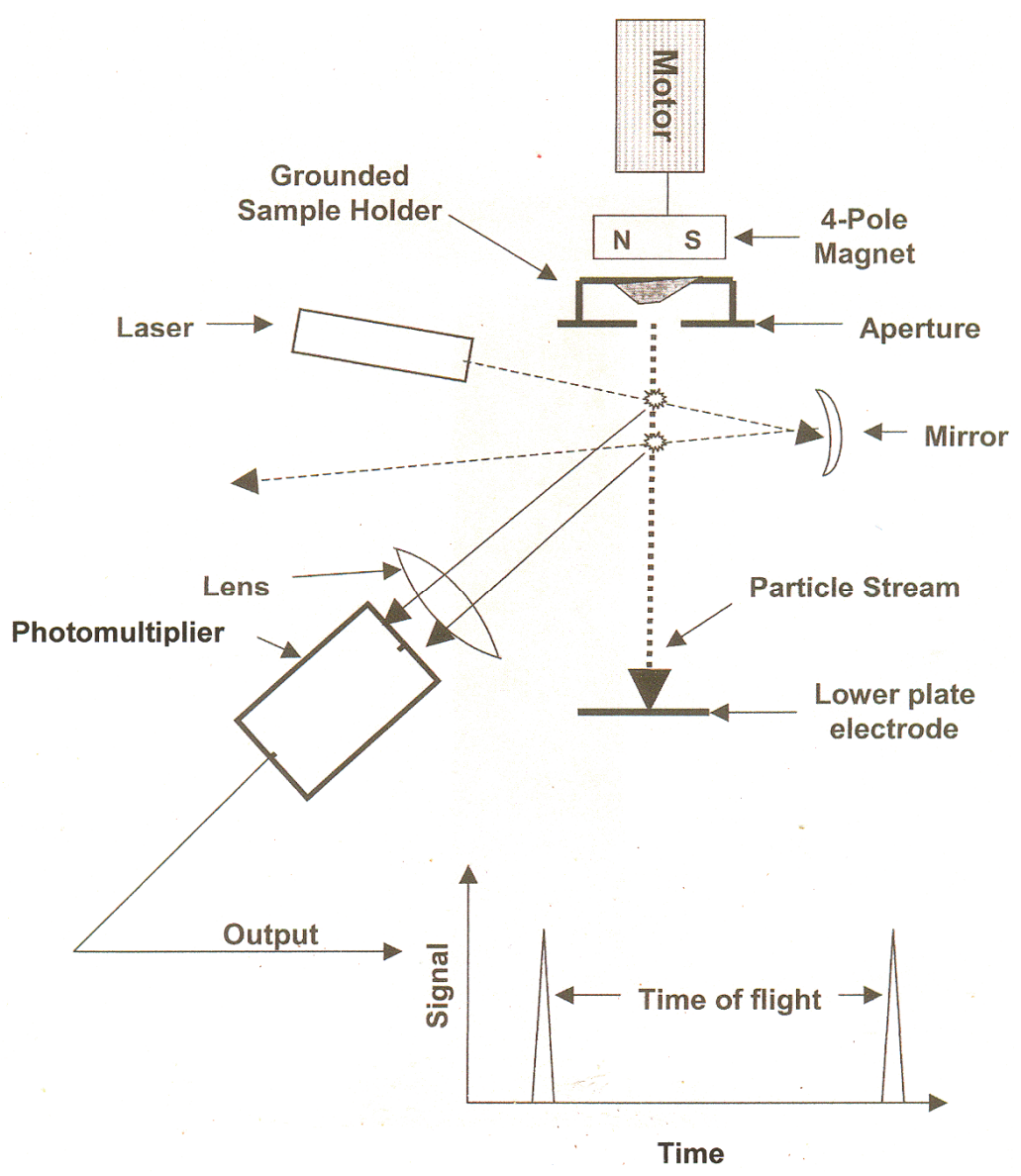

Figure 1. Diagram of the instrument.

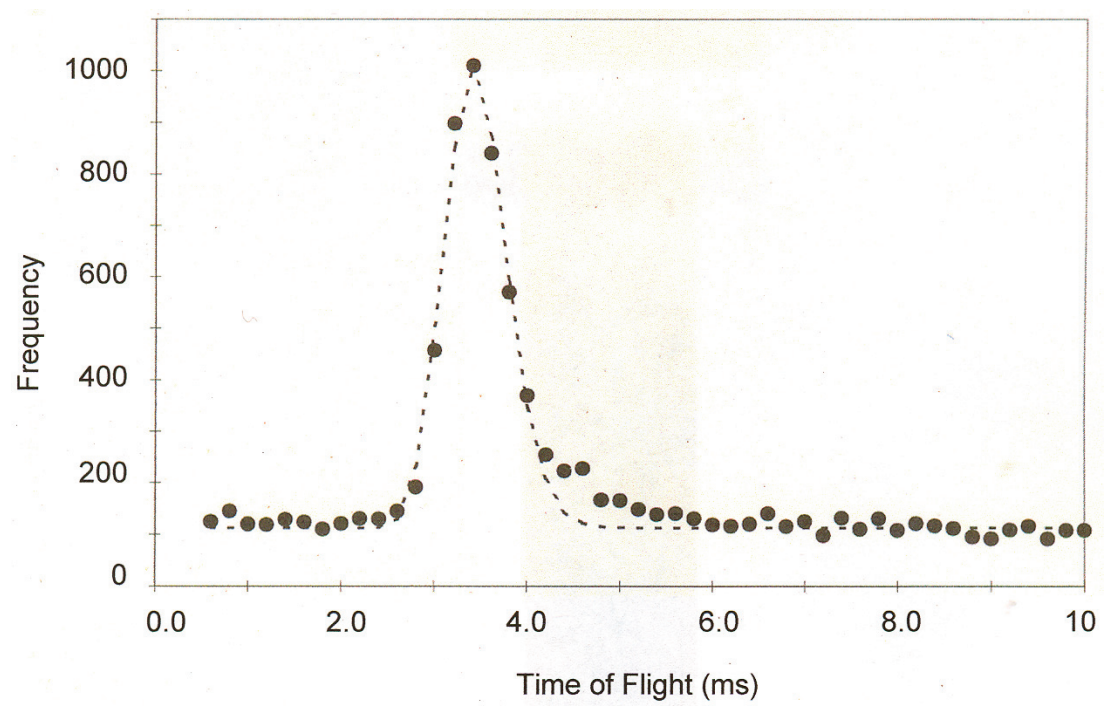

Figure 2. Time of flight distribution for a positively charging toner. 


\section{Instrument Design and Operation}

In the instrument described in this paper, the terminal velocity is determined by measuring the time of flight of the particles between two laser beams. Fig. 1 is a diagram of the instrument. To introduce charged toner particles into the instrument, a small developer sample (1 -5 mg) is placed in a grounded sample holder and held in place by a 4-pole magnet. A grounded metal aperture with a $1 \mathrm{~mm}$ hole is placed in contact with the sample cup. The magnet is rotated at 10-1000 rpm to agitate the developer. Toner is pulled off the developer by an electric field $(500-5000 \mathrm{~V} / \mathrm{cm})$ applied between the sample holder and the lower plate electrode, which is approximately $3 \mathrm{~cm}$ from the sample cup. This generates a beam of particles approximately $1 \mathrm{~mm}$ in diameter. Terminal velocity of the charged toner particles in the ambient air is determined by measuring the time of flight of the particles between two laser beams separated by approximately $1 \mathrm{~mm}$. The second beam is generated by reflecting the first beam from a He-Ne laser back through the sampling space with a mirror. Separation between the beams can be varied by adjusting the mirror angle. When a particle passes through a laser beam, a pulse of scattered light is detected with a photomultiplier. An A/D converter is used to convert the analog pulse from the photomultiplier to a digital signal. The time of the center of the pulse is determined by the point at which the derivative of the signal changes sign. Because many particles may be passing through the first beam before the first particle passes through the second beam, it is necessary to use a correlation technique to find the distribution of times of flight of the particles. This is done by calculating the time difference between a given pulse and ten succeeding pulses and plotting the resulting distribution. An example of a time of flight distribution is shown in Fig. 2.

Time of flight can be converted to the charge to diameter ratio, $\mathrm{q} / \mathrm{d}$, of the particles as follows:

The net force on a charged particle in an electric field is given by

$$
\text { Net force }=\text { Electrostatic Force }- \text { Drag Force }
$$

The electrostatic force is given by the product of the charge on the particle, $\mathrm{q}$, and the electric field strength, E. The drag force is given by Stokes law:

$$
\text { Drag Force }=3 \pi \eta \mathrm{d} \mathrm{v}
$$

In Eq. 2, $\eta$ is the viscosity of the air, $\mathrm{d}$ is the particle diameter, and $\mathrm{v}$ is the velocity of the particle. At terminal velocity the net force is zero and the electrostatic force equals the drag force:

$$
\mathrm{q} \mathrm{E}=3 \pi \eta \mathrm{d} \mathrm{v}
$$

The ratio of the charge on the particle to its diameter is given by:

$$
\mathrm{q} / \mathrm{d}=(3 \pi \eta) \times \mathrm{v} / \mathrm{E}
$$

Time of flight distributions can be converted to $\mathrm{q} / \mathrm{d}$ distributions using Eq. (5):

$$
\mathrm{q} / \mathrm{d}=(3 \pi \eta) \times(\mathrm{s} / \Delta \mathrm{t}) \times \mathrm{L} / \mathrm{V}
$$

In Eq. $5 \mathrm{~s}$ is the separation of the laser beams $(\approx 1 \mathrm{~mm}), \Delta \mathrm{t}$ is the difference in time between the pulse created by the particle as it passes through the first beam and the pulse created by the particle passing through the second beam, L is the spacing between the developer sampler and the collector plate $(1.6 \mathrm{~cm})$, and $\mathrm{V}$ is the applied potential in volts.

A table of time of flights was created with a BASIC program from the stored digital signal from the A/D board. (Only signals above a preset threshold were stored to eliminate noise and minimize the amount of data to be stored.) Correlation analysis and plotting of histograms were done using Microsoft EXCEL. The q/d distributions were plotted with either a linear or logarithmic scale for q/d on the X-axis. The advantage of a logarithmic scale is that the breadth of the distributions is independent of the mean of the distribution so that the width of distributions of materials with different $\mathrm{q} / \mathrm{d}$ can be compared visually. The linear scale accentuates small differences in distributions making it easier to identify more subtle differences between distributions.

\section{Results}

The data presented in this section are intended to illustrate the capability of the equipment.

Fig. 2 shows time of flight measurements made with a commercially available positively charging black developer using a small Fe-Sr ferrite carrier. The developer was tribocharged charged for 600s in a magnetic stirrer at $100 \mathrm{rpm}$. The potential applied to the lower plate electrode to develop the toner was 
$-2000 \mathrm{~V}$. The line in the figure is a log-normal distribution fitted to the data. (Mean $=3.00 \mathrm{~ms}$ Std. Dev.. $=1.17 \mathrm{~ms})$

Fig. 3 is an illustration of a q/d distribution determined for approximately 20,000 particles of a chemically prepared magenta toner charged with a silicone-coated Fe-Sr ferrite carrier. The baseline resulting from random intervals between particles entering the system amounts to about 100 particles per channel. The peak resulting from the time of flight of the particles between the two beams is approximately 50 times greater than the background. The ratio of the peak maximum to the standard deviation of the background is typically between 10 and 100 .

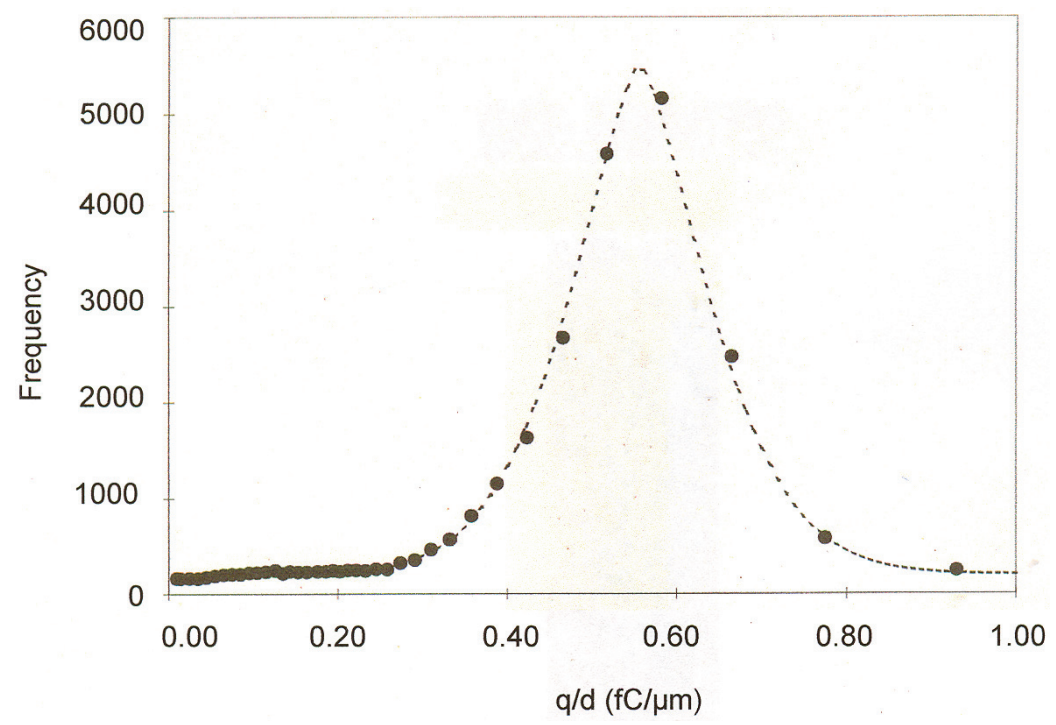

Figure 3. Q/d distribution for approximately 20,000 particles.

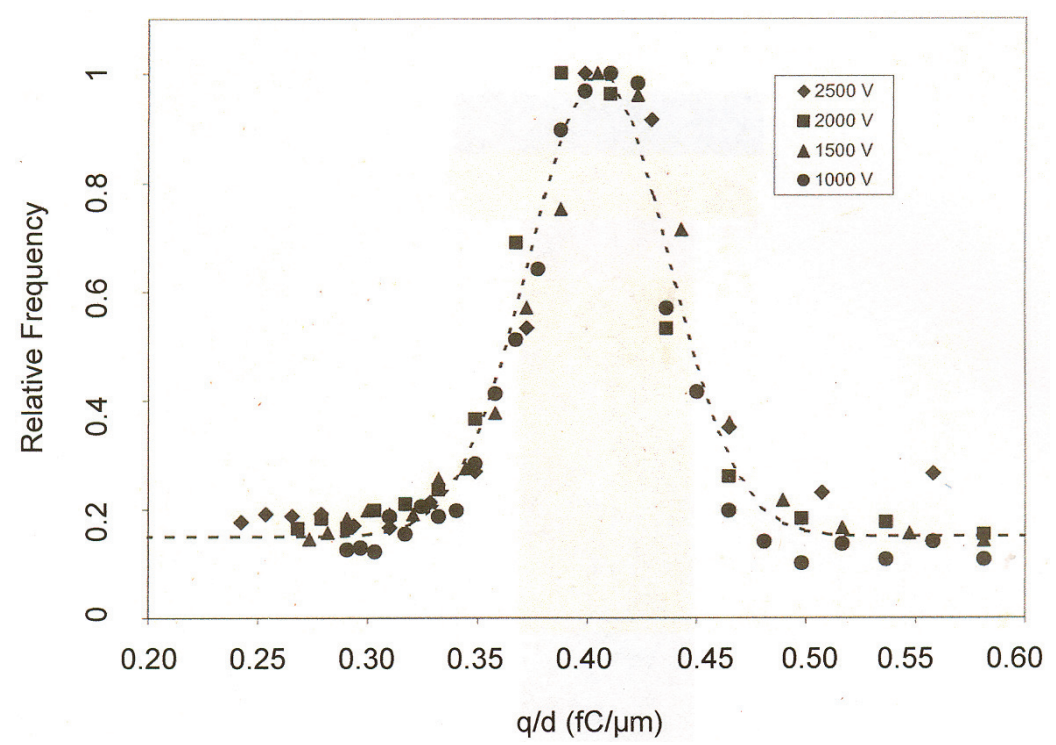

Figure 4. Q/d distributes taken at a series of applied potentials.

A set of experiments was performed to explore the effect on the measurement of the magnitude of the electric field applied between the aperture and the lower plate electrode. A black styrene acrylic based toner was tribocharged using a poly (vinylidene fluoride) coated Fe-Sr ferrite carrier by stirring for 600s at $100 \mathrm{rpm}$ in a magnetic stirrer. Fig. 4 shows that $\mathrm{q} / \mathrm{d}$ measurements taken at several different negative potentials applied to the lower plate electrode superimpose well. Fig. 5 is a plot of the reciprocal of the 
mode of the time of flight distributions for the same series of measurements shown in Fig. 4. The plot is linear with a correlation coefficient, $\mathrm{R}^{2}$, of 0.99 . This shows that the velocity, which is proportional to the reciprocal of the time of flight, is a linear function of the applied potential in agreement with Eq. (3).

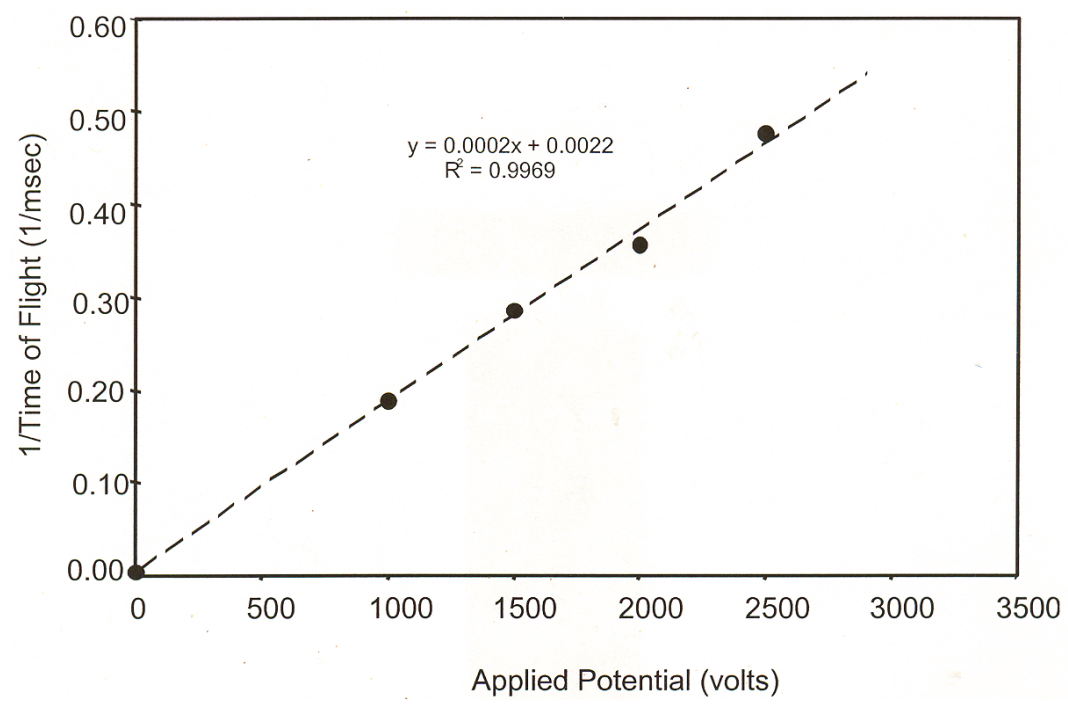

Figure 5. Reciprocal of the modes of the time of flight distributions for the same series of measurements shown in Fig. 4.

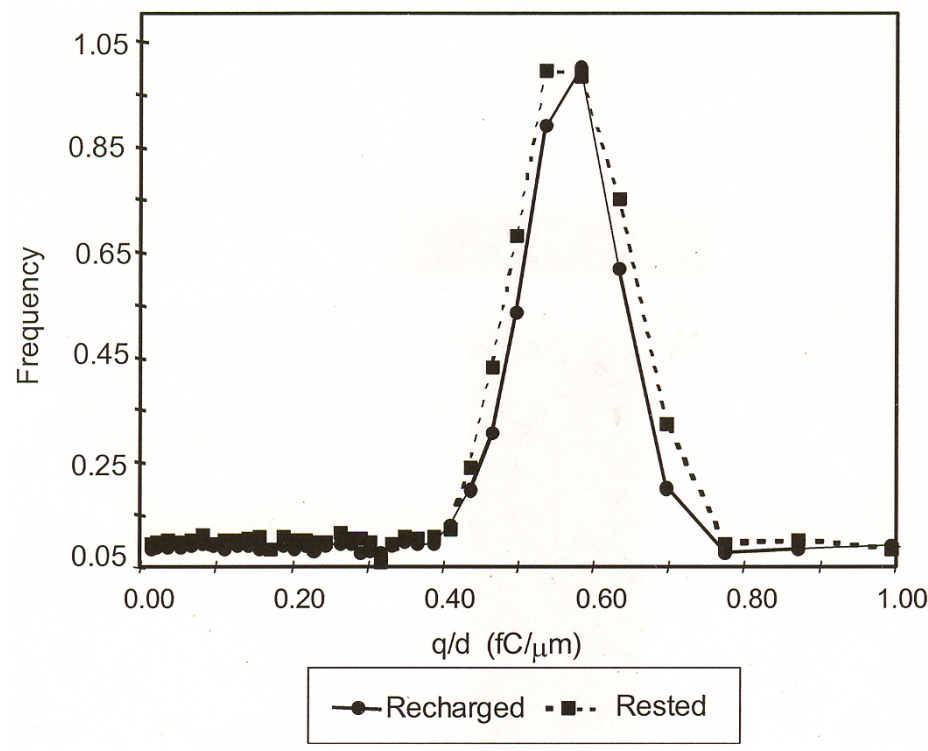

Figure 6. Comparison of $\mathrm{q} / \mathrm{d}$ distributions of a tribocharged toner sample after resting for several weeks and after recharging.

Fig. 6 compares the $\mathrm{q} / \mathrm{d}$ distribution of styrene acrylic based black toner rested for several weeks after charging for one hour at $2000 \mathrm{rpm}$ on a magnetic stirrer with the $\mathrm{q} / \mathrm{d}$ distribution of the same sample recharged for 30s in an AC magnetic field. It can be seen that after recharging the mean of the distribution remains approximately the same, while the distribution narrows slightly.

Fig. 7 illustrates charging behavior of $50 \mathrm{mg} / \mathrm{g}$ fresh toner added to the fully charged developer from Fig. 6 . The added toner peak, which has the lower $\mathrm{q} / \mathrm{d}$ in the figure moves to the right as it charges. The peak of the originally charged toner moves slightly to lower $\mathrm{q} / \mathrm{d}$ indicating that charge is donated from the charged toner to the added toner. (Compare Fig. 6 to Fig. 7.) After 600s of mixing, the two peaks have 
merged at a q/d value slightly lower than before addition of toner. The lower charge is expected since the toner concentration increases when toner is added.

It is also possible to obtain q/d distributions from toner charged with larger, magnetically soft carriers, e.g. $100 \mu \mathrm{m}$ iron or stainless steel. Fig. 8 compares the $\mathrm{q} / \mathrm{d}$ distributions of a black styrene acrylic toner charged on a $100 \mu \mathrm{m}$ Kynar coated stainless steel carrier with the same toner charged on a $30 \mu \mathrm{m}$ Kynar coated ferrite carrier. The distribution is broader on the stainless steel carrier.
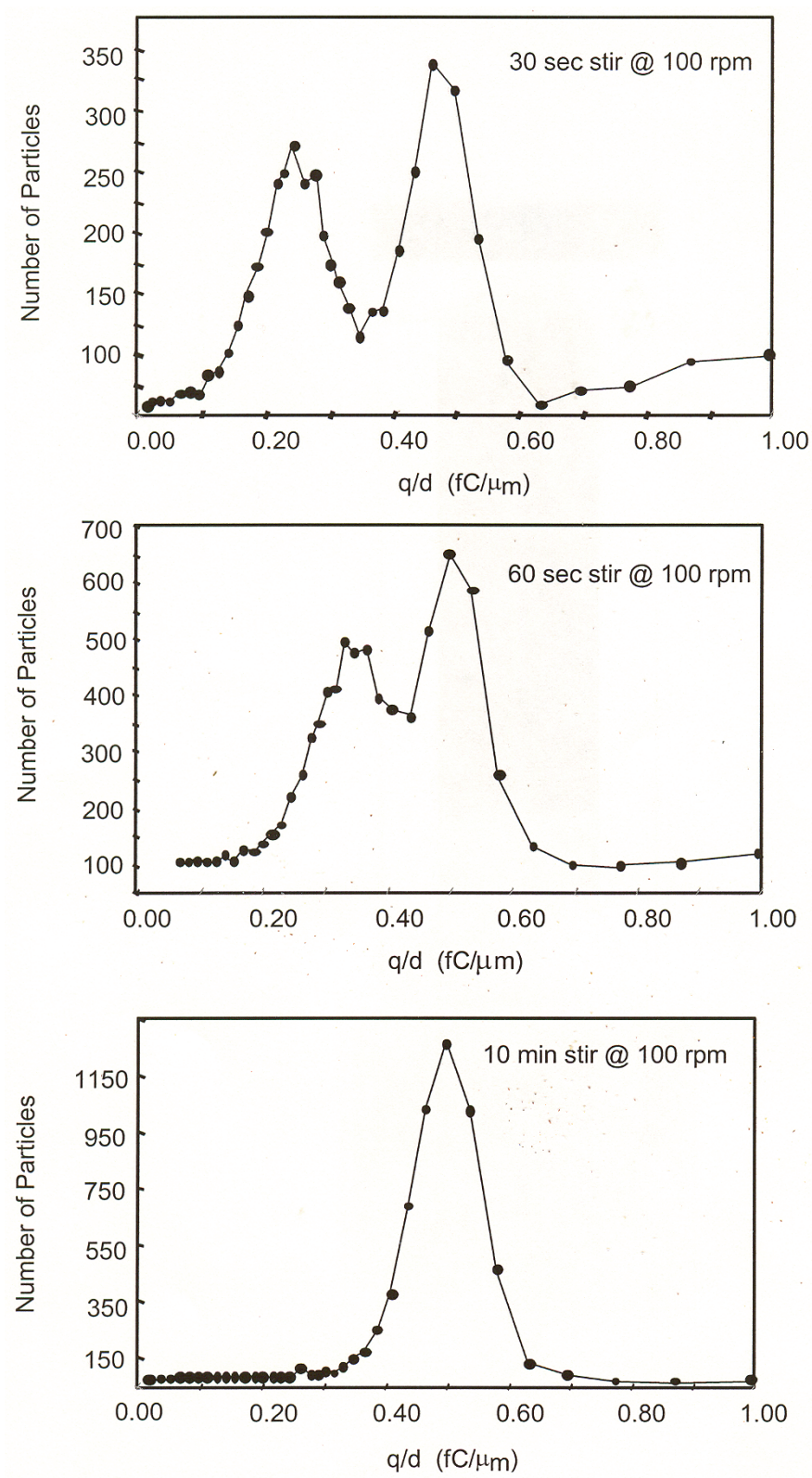

Figure 7. Q/d distributions of the sample shown in Fig. 6 as a function of charging time after the addition of a small amount of uncharged toner. The peak for the added toner is on the left, and merges with the $\mathrm{q} / \mathrm{d}$ peak of the original toner as the developer is charged. 

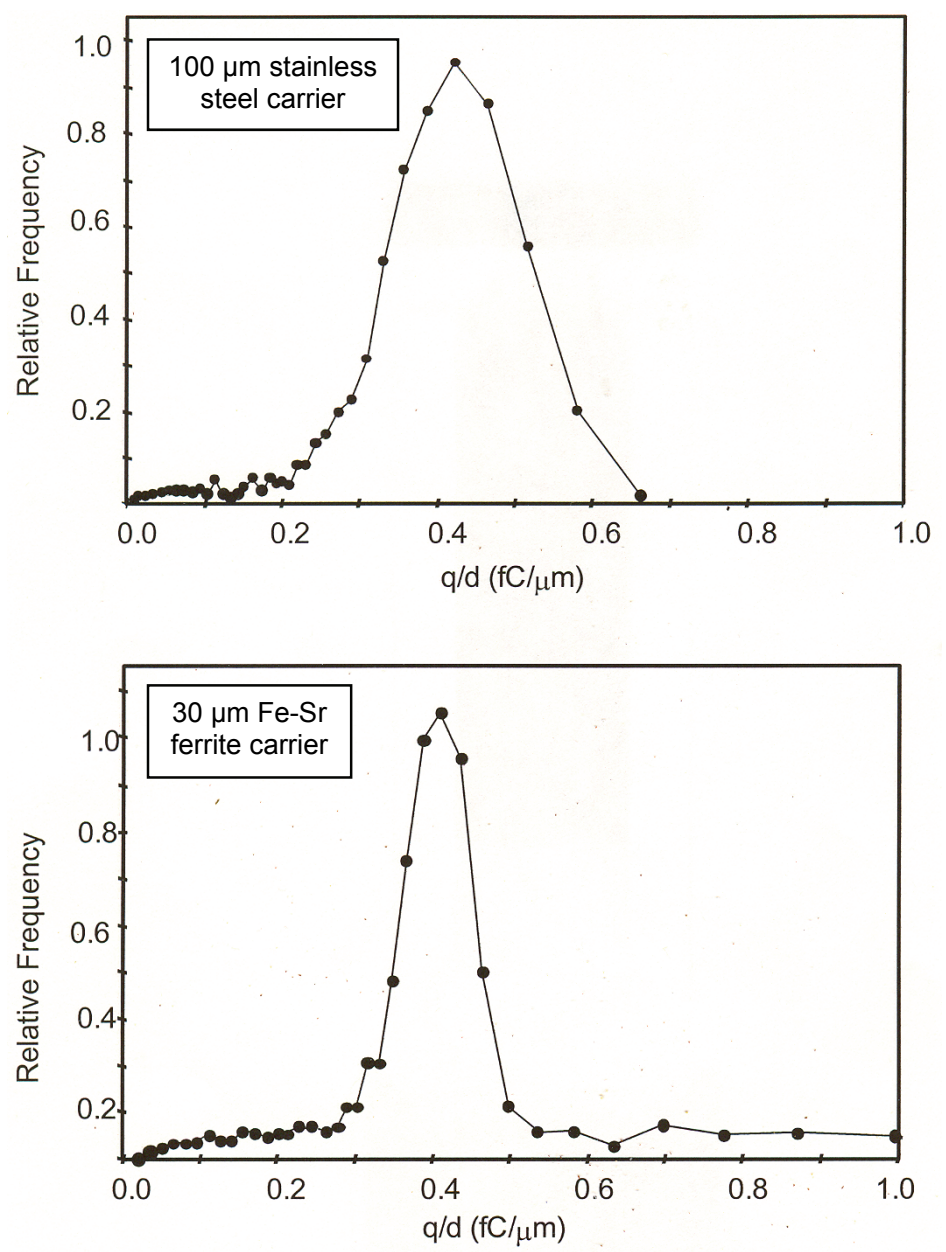

Figure 8. Comparison of the $\mathrm{q} / \mathrm{d}$ distributions of the same toner tribocharged using two different carriers.

\section{Discussion and Conclusions}

A time-of-flight method of determining $\mathrm{q} / \mathrm{d}$ distributions of toner has been tested. The method gives results, which are similar to those found by other workers. That is, the values of $\mathrm{q} / \mathrm{d}$ are in the range found by others $(0.0 \pm 2 \mathrm{fC} / \mathrm{\mu m})$. [7,8]. The distributions are independent of the measuring electric field, as they should be, and they are reproducible. Toner $\mathrm{q} / \mathrm{d}$ distributions can be obtained from developers made with small permanently magnetized ferrite carriers and larger magnetically soft metal or ferrite carriers.

The main advantages of this instrument are that it is easy to operate, has rapid turnaround (approximately $15 \mathrm{~min}$. per sample), and $\mathrm{q} / \mathrm{d}$ distributions can be obtained without a secondary optical scanning step as required by the Epping $\mathrm{q} / \mathrm{d}$ meter or the quantitative charge spectrometer of Gee et al $[1,2,7,8]$. No particle size information can be obtained with this instrument as is also the case with the Epping $\mathrm{q} / \mathrm{d}$ meter. Both particle size and charge are obtained for individual particles by the E-SPART analyzer and the Gee spectrometer $[4,5,8]$. However, this is not a major disadvantage since $\mathrm{q} / \mathrm{d}$ distributions are sufficient for most applications. A more serious disadvantage is that low charged and uncharged particles cannot be detected because they are not significantly influenced by the electric field, and they drift out of the measuring space.

This instrument should be applicable to measuring $\mathrm{q} / \mathrm{d}$ of any type of small particle, if a suitable sampling system is employed. 


\section{References}

1. q/d-meter, Epping GmbH, www.epping-pes.de/

2. Reinhold H. Epping and Andreas Kuettner, J. Electrostatics Volume 55, July 2002, Pages 279-288

3. Nicholas Hesse et al., Polymers 2019, 11(4) pg. 609.

4. E-SPART analyzer, Hosokawamicron Corporation, https://hosokawamicron.co.jp/en/machines/172.

5. Mazumder, M.K.. (1993). KONA Powder and Particle Journal. 11. 105-118. 10.14356/kona.1993013

6. D. Sami et al. Pharmaceutical Development and Technology, Vol. 12, No. 1 pp. 35-41.

7. R. B. Lewis, R. F. Koehler and E. W. Connors, Charge Spectrograph, U. S. Patent 4,375,673, March 1, 1983.

8. G. P. Gee et al in Recent Progress in Toner Technologies, G. Marshall, Ed. IS\&T, pp. 195-202. 